\title{
La décentralisation des politiques culturelles
}

The Decentralisation of Arts Policies

\section{Cécile Doustaly}

\section{(2) OpenEdition}

1 Journals

Édition électronique

URL : http://journals.openedition.org/rfcb/3426

DOI : $10.4000 /$ rfcb.3426

ISSN : 2429-4373

Éditeur

CRECIB - Centre de recherche et d'études en civilisation britannique

\section{Édition imprimée}

Date de publication : 1 avril 2005

ISBN : 2-911580-20-6

ISSN : 0248-9015

\section{Référence électronique}

Cécile Doustaly, "La décentralisation des politiques culturelles », Revue Française de Civilisation Britannique [En ligne], XIII-2 | 2005, mis en ligne le 01 avril 2005, consulté le 10 avril 2020. URL : http:// journals.openedition.org/rfcb/3426; DOI : https://doi.org/10.4000/rfcb.3426

Ce document a été généré automatiquement le 10 avril 2020.

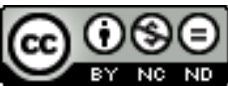

Revue française de civilisation britannique est mis à disposition selon les termes de la licence Creative Commons Attribution - Pas d'Utilisation Commerciale - Pas de Modification 4.0 International. 


\title{
La décentralisation des politiques culturelles
}

\author{
The Decentralisation of Arts Policies
}

Cécile Doustaly

1 En 1946, les partis politiques se sont accordés sur la mise en place d'un modèle bureaucratique permettant un soutien de la culture dit paternaliste car il s'attachait à la démocratisation des arts d'élites. Le ministère de tutelle finançait à distance (at arm's length) par l'intermédiaire de l'Arts Council of Great Britain, organisme nongouvernemental dirigé par des experts non élus issus des milieux dirigeants et géré par une administration indépendante de la fonction publique. Les subventions de l'Arts Council ont souvent été bien accueillies par les collectivités locales, nous verrons cependant que les relations entre ces deux sources de soutien qui n'ont officiellement aucun pouvoir l'une sur l'autre ont toujours été complexes. Une décentralisation, si elle respecte ce modèle encore en place, ne peut donc se faire au profit des localités, mais suppose alors un déficit de démocratie locale.

2 En Angleterre, la décentralisation culturelle fonctionne aujourd'hui au niveau régional et local. La régionalisation est une force qui pèse sur toutes les agences nongouvernementales d'exécution (executive Non-Departmental Public Bodies) actives dans le soutien aux arts. Resource (musées) et le National Film Council, créés depuis peu, jouent le jeu de la délégation des pouvoirs à leurs agences régionales ${ }^{1}$ alors que la réforme de l'Arts Council England (ACE), qui subventionne les arts de la scène et les arts visuels, a été très controversée car elle aboutissait pour certains à une centralisation. Cet article se propose, en se concentrant sur l'ACE, d'analyser la nature des nouveaux rapports entre le double niveau national et les trois échelons décentralisés ainsi que la rhétorique mise en place pour défendre cette politique. La décentralisation tient-elle ses promesses? S'accompagne-t-elle d'une dévolution du pouvoir au profit des agences régionales de l'Arts Council ou des localités ${ }^{2}$ ? Permet-elle une meilleure intégration des citoyens à l'élaboration des politiques culturelles?

3 L'étude de cas de Preston ${ }^{3}$ apportera un éclairage sur la décentralisation culturelle dans cette agglomération de 129000 habitants, capitale culturelle, commerciale et 
administrative du Lancashire ${ }^{4}$. La ville a obtenu le statut de City en 2002 mais elle souffre de la proximité de Liverpool et de Manchester. La mairie, travailliste, s'appuie notamment sur la culture pour s'imposer dans la région et pour soutenir les quelques quartiers défavorisés. L'ensemble de l'administration et le département Regeneration, Community and Leisure ont été jugés d'un bon rapport qualité-prix lors de l'inspection de l'Audit Commission en 2003, ce qui fait de Preston un exemple adapté pour mesurer les exigences du ministère en terme de politique culturelle ${ }^{5}$.

\section{La décentralisation culturelle : définitions}

4 Les trois modèles administratifs présentés en introduction de ce numéro s'appliquent imparfaitement à la culture, dont le statut de service public est particulier. L'Arts Council compte parmi les premiers organismes non-gouvernementaux introduisant une décentralisation fonctionnelle des missions ministérielles ${ }^{6}$. Comme dans d'autres services publics, cependant, de nouveaux modèles de décentralisation viennent complexifier l'alternative classique entre «transfert des ressources disponibles » et "dévolution des responsabilités »".

5 La décentralisation des pouvoirs de l'Arts Council effectuée en 2002 était préconisée dès les années 1970. On distinguait déjà les trois types de décentralisation culturelle qui se juxtaposent aujourd'hui. Dans le premier, le centre utilise la décentralisation comme le moyen le plus à même de mettre en place sa politique nationale. Les deux autres types de décentralisation ont une fin en soi. Elle est géographique lorsqu'on procède au transfert de pouvoir aux régions afin de limiter la domination d'une culture dictée du centre. M. Green, l'un des premiers à avoir étudié le sujet, ne trouve pas éclairant de définir l'Angleterre dans son ensemble comme le centre et lui préfère l'opposition entre métropoles et marges. Il explique le peu d'attrait de la décentralisation culturelle en Angleterre par le manque de poids des cultures régionales par comparaison aux cultures variées qui cohabitent dans les villes. Le troisième type de décentralisation implique le transfert de pouvoir d'une culture dominante à une culture minoritaire, ou démocratie culturelle ${ }^{8}$. Plus récemment, d'autres analystes ont distingué décentralisation culturelle (combattre les inégalités d'accès à la culture), fiscale (distribution juste et suffisante de fonds), et politique (mettre la gouvernance en pratique) $)^{9}$.

6 Tant du côté des chercheurs que des pouvoirs publics, la décentralisation de l'offre culturelle nationale (souvent les high arts) a rarement été préconisée à cause du coût et de l'objectif premier des productions: la qualité. À l'inverse, dès 1968, certains ont recommandé la décentralisation des politiques culturelles locales, de préférence à une agence régionale ${ }^{10}$. La décentralisation au profit des localités, rarement envisagée, aurait supprimé le principe d'autonomie qui a néanmoins été ensuite mis à mal par leur représentation au sein des Regional Arts Associations.

7 La notion de démocratie culturelle a été la source de débats houleux sur la définition de l'art. Si l'on se place dans une logique de service public, il semble cependant que les besoins de certains groupes ont longtemps été ignorés, souvent parce qu'on considérait qu'ils étaient satisfaits par l'offre culturelle commerciale. Nous verrons qu'il reste difficile pour les échelons décentralisés de concilier cultures minoritaires et critères traditionnels d'excellence, longtemps défendus par l'Arts Council. Bien que le lien direct entre décentralisation et démocratie culturelle ne soit pas automatique, on 
attend souvent de la décentralisation culturelle davantage de citoyenneté, de transparence politique et la valorisation de l'identité locale. Le point d'équilibre permettrait de sauvegarder stratégie et qualité nationales tout en soutenant au niveau local l'autonomie, l'initiative et la pratique :

The advantages of the centralising model seem to be a guarantee of control, perhaps of standards, and a consistency of approach; it may also reflect greater governmental commitment to culture. The advantages of decentralisation are a closer correlation between provision and local needs, greater opportunity for cultural diversity and an empowerment of local action and participation. ${ }^{11}$

8 L'évolution du concept de culture remet ainsi en contexte le soutien en faveur de la décentralisation. Peter Hewitt, l'actuel directeur général de l'Arts Council, décrit la nouvelle politique de l'organisme davantage axée sur les usagers : entre refus de dicter une culture officielle et écoute des besoins de tous. On observe ainsi la complexification du rôle de l'ACE comme pourvoyeur de service public et outil de démocratie locale : ' The open space for artistic expression is expanding. It is increasingly difficult in a more democratic, self-organising, less deferential and more educated society to prescribe any one particular role for art to play [...] The Arts Council's fundamental role [...] is to promote exploration through art. ${ }^{12}$ Sir Christopher Frayling, actuel directeur de l'Arts Council, incarne bien la distance parcourue par l'organisme depuis sa création. Recteur du Royal College of Art, c'est néanmoins un spécialiste de culture populaire qui soutient la diversité culturelle ${ }^{13}$. Au niveau local, on verra que cette conception large des arts dominait déjà car ils ont traditionnellement été intégrés à des objectifs multiples (éducation, insertion, santé). Les gouvernements de Tony Blair semblent donc avoir fait converger les politiques de ces deux sources de soutien, ce qui facilite leur collaboration.

\section{Historique du modèle de soutien public aux arts}

9 La politique culturelle d'après-guerre était réactive, reposant sur le soutien à des institutions peu rentables ${ }^{14}$. L'Arts Council a longtemps privilégié Londres et les grandes institutions artistiques, surtout après la fermeture de ses bureaux régionaux en $1956^{15}$. La culture en province était soutenue de façon très inégale, les collectivités locales n'ayant pas d'obligation statutaire de la soutenir (on les y autorisa de façon discrétionnaire en 1948). De nombreuses localités ne voulaient pas ou ne savaient pas comment soutenir les arts. Ceci explique le développement du rôle de l'Arts Council, soit directement, soit par l'intermédiaire d'agences régionales. Les localités travaillistes ont toujours dépensé davantage pour la culture, en partie parce que le Parti travailliste dirigeait les grandes métropoles. Les conservateurs souhaitaient limiter les dépenses culturelles, considérées comme une marge de l'État-providence. Les libéraux prenaient généralement une position médiane.

Ce consensus d'après-guerre a été remis en cause par le gouvernement Wilson, période souvent désignée comme l'âge d'or de l'Arts Council, qui s'intéressa davantage aux arts du spectacle, à la province, et à une culture plus diverse et plus novatrice. Cette nouvelle orientation a établi un modèle de démocratie culturelle visant à intégrer tous les citoyens dans une conception plus large de l'art, ce qu'a illustré la naissance puis le financement des community arts. À partir de 1966, certaines responsabilités de l'Arts Council, accompagnées de fonds, ont été dévolues à des Regional Arts Associations (RAAs) pour faire taire les accusations de centralisme et adapter l'offre aux régions. 
Certaines RAAs ont été créées par des organisations régionales, d'autres par les collectivités locales. L'Arts Council a choisi de collaborer sans abandonner aux RAAs et aux collectivités son rôle d'évaluation de la qualité des productions artistiques. Il soutenait seul les grandes institutions qui préféraient avoir affaire directement à lui, mais il a progressivement délégué certaines fonctions que les RAAs étaient mieux à même de gérer : les activités à visée sociale ou éducative comme l'animation culturelle et les community arts. Ces besoins, mieux connus des collectivités, comptaient souvent parmi leurs priorités puisqu'ils étaient liés à leurs fonctions statutaires (éducation, santé). L'Arts Council refusait par ailleurs, sans augmentation de son allocation, de s'investir dans ces domaines. En 1976, Lord Redcliffe-Maud déclarait ainsi dans le rapport où il introduisait l'expression arm's length : 'if Regional Arts Associations [...] did not exist [...] we would have to invent them ${ }^{16}$.

11 Au début des années 1970, les incohérences du système décentralisé étaient flagrantes. L'Arts Council était en faveur d'un partenariat dénué de délégation des pouvoirs ${ }^{17}$. Le rôle des RAAs devait donc se limiter à celui d'exécutant de la politique nationale. Les RAAs, cependant, n'avaient pas de compte à rendre à l'Arts Council quant à leur choix de subventions, dont le montant global et la nature connaissaient de fortes variations. De plus, loin d'être augmentée, la participation financière des collectivités locales avait chuté de $52 \%$ à $15 \%$ entre $1963-64$ et $1978-79^{18}$ sans que leur poids au sein des RAAs soit amoindri :

C'est avec insistance que l'Arts Council réclame une participation plus substantielle des collectivités locales au financement des Associations; il constate que celles-ci reçoivent [de l'Arts Council] $70 \%$ du total de leurs ressources alors que dans l'élaboration de leur politique, elles sont surtout sous l'influence des pouvoirs locaux ${ }^{19}$.

Durant les années 1980, les politiques culturelles ont évolué vers des positions partisanes. Margaret Thatcher raconte dans ses mémoires : 'I was not convinced that the state should play Maecenas. [...] Moreover the "state" in this case comes to mean the vested interests of the arts lobby. ${ }^{20}$ Le niveau national privilégiait le patrimoine et le financement privé alors que le Greater London Council (GLC) exprimait l'opposition travailliste par une stratégie d'octroi des droits (empowerment strategy) en faveur des communautés et par les communautés : 'The arts policies of the GLC [...] understood the arts in a political fashion. The intention of an arts policy was no longer to simply support the traditional "high" arts [...] to provide disadvantaged groups within the community with their own voice. ${ }^{21}$. Le GLC a également développé l'utilisation des arts à des fins économiques. Nous verrons que ces spécificités se retrouvent dans la politique et dans la rhétorique de l'actuel gouvernement travailliste.

La relation conflictuelle entre les RAAs et l'Arts Council s'inscrit donc dans un réseau plus large de tensions structurelles qui n'allaient être que partiellement résolues par les réformes des années 1980 et 1990. En 1989, le Wilding Report faisait à nouveau le bilan de vastes disparités dans la méthode de répartition des subventions selon les régions et a abouti en 1991 au remplacement des douze RAAs par dix Regional Arts Boards (RABs), entreprises caritatives indépendantes bien que financées en majorité par l'Arts Council ${ }^{22}$. Un tiers de leurs membres devait être nommé par les collectivités locales, les autres étant issus du milieu local des affaires ou de la culture. Les RABs ont reçu la responsabilité de subventionner les organisations auparavant gérées par l'Arts Council, à l'exception des compagnies nationales. 
14 En 1992, la création du premier ministère principalement consacré à la culture, le Department for National Heritage, suivie par sa refonte en un Department for Culture, Media and Sport (DCMS) en 1997, signale la reprise de l'initiative politique par le gouvernement central, dans un contexte de recherche de rentabilité des services publics. Après une période d'indépendance durant laquelle il a mené assez librement ses choix de politique culturelle au sein de contraintes budgétaires, l'Arts Council a ainsi dû se plier aux directives plus contraignantes de son ministère de tutelle ${ }^{23}$, bien qu'il soit resté libre de décider à quels organismes allouer les fonds. Les organisations artistiques subventionnées ont subi par ricochet des pressions similaires de la part de l'Arts Council. C'est donc à une contractualisation des rapports et à une pression grandissante du centre que l'on a assisté durant les années 1990, malgré la diminution d'environ 9 \% des dépenses totales en faveur de l'art entre 1979 et 2004. Les travaillistes ont imposé des objectifs précis en augmentant les fonds alloués à l'Arts Council de $120 \%$ en livres courantes entre $1997-98$ et 2005-06 (hors loterie nationale) ${ }^{24}$.

15 Cette politisation et cette centralisation du soutien à la culture ont connu des détracteurs en Angleterre, pour lesquels l'intervention néfaste de la politique dans ce domaine a été prouvée par l'échec retentissant du Dôme ${ }^{25}$. Soucieux de l'opinion publique, le gouvernement de Tony Blair a alors tenté de rationaliser les dépenses et de rééquilibrer l'offre en faveur des régions. Depuis 1999, les Regional Development Agencies ont été complétées par des Cultural Consortiums et des agences régionales d'un Arts Council England réformé. Quelles formes la décentralisation pouvait-elle prendre sachant que la structure existante des pouvoirs intègre un organisme nongouvernemental en sus des niveaux nationaux et locaux ? Cette décentralisation étaitelle compatible avec un ministère relativement dirigiste?

\section{La décentralisation de l'Arts Council of England}

Contrairement à l'Arts Council, le ministre peut toujours se cacher derrière le principe d'autonomie pour éviter de porter la pleine responsabilité des décisions de l'organisme devant le Parlement. Cependant, il semble que les réformes correspondent aux périodes durant lesquelles les critiques ont atteint des sommets. En 1994, les pouvoirs de l'Arts Council of Britain furent dévolus aux régions, ce qui a abouti à la création d'Arts Councils indépendants pour l'Angleterre, l'Écosse et le Pays de Galles, précédant ainsi la dévolution des Parlements en $1997^{26}$. En Angleterre, il était plus difficile pour les partisans de la décentralisation d'arguer de fortes différences régionales au sein de la culture subventionnée. Ils ont davantage insisté sur la reconnaissance de besoins locaux pour justifier le rééquilibrage des fonds et des pouvoirs ${ }^{27}$. Parallèlement à ces demandes, les organisations artistiques ont exprimé leur mécontentement face à la proportion du soutien à la culture dépensé en administration. Bien que les fonds aient augmenté de façon significative depuis la création de la loterie nationale en 1993 et après le budget travailliste de 1998, la charge de travail et les coûts administratifs de l'Arts Council comme du ministère se sont accrus. Les coûts d'administration se sont également alourdis à cause d'un premier mouvement de décentralisation des fonds au profit de petites associations (Lottery for All, Regional Arts Lottery Programme, Voluntary Arts Network) ${ }^{28}$. La réforme de l'ACE en 2002 a-t-elle répondu aux attentes divergentes exprimées à l'extérieur comme à l'intérieur du gouvernement? 

travailliste, qui a été choisi par le gouvernement pour moderniser et démocratiser l'organisme, avec succès si l'on en croit The Guardian ${ }^{29}$. Son projet de réforme, présenté comme une décentralisation, consistait à remplacer l'Arts Council of England et les dix RABs avec lesquels il coopérait par un organisme unique comportant un bureau national et neuf agences régionales correspondant aux limites géographiques des Regional Development Agencies ${ }^{30}$. Gerry Robinson a déclaré sa foi en la délégation :If you make every decision and take control of the whole thing, you end up with what I call the Thatcher Phenomenon and nobody of any capacity or weight will stay in that environment ${ }^{31}$. officiellement, l'objectif était de créer un système de subventions plus simple et plus transparent tout en réalisant des économies d'administration qui pourraient ensuite être réinvesties dans les arts $^{32}$.

2001, lorsque cette réforme a commencé à être discutée, les partis d'opposition et la majorité des localités s'y sont opposés car elle allait selon eux à contre-courant de l'élan gouvernemental de décentralisation. Ils redoutaient que l'ACE ne décentralise les fonds tout en centralisant les choix politiques, privant ainsi les régions de leur indépendance passée ${ }^{33}$. Les libéraux démocrates ont ainsi présenté un projet inverse aux élections de 2001: 'We would devolve all funding decisions, except those relating to the national institutions. [...] Making funding decisions nearer to communities will foster greater variety and diversity than one national body ${ }^{\prime 34}$. Ken Livingstone et l'assemblee de Londres se sont élevés contre la disparition du London Arts Board, exigeant la création d'un bureau intégré à la Greater London Authority :

It will take power away from London and the people of London and leave funding decisions [...] in the hands of an unaccountable national quango. [...] If the Arts Council really believes in decentralisation - as its paperwork suggests - then surely the best approach would be to hand over responsibility for funding the arts in London to the GLA..$^{35}$

Le monde artistique n'a pas présenté un front uni face à la réforme. Certaines institutions artistiques redoutaient une décentralisation en faveur d'agences régionales, craignant que les collectivités locales n'y aient davantage de pouvoir et ne se montrent moins éclairées, plus rétives à l'art pour l'art, plus frileuses face aux coûts élevés. Cette tendance, constatée depuis 1946, est reconnue dans le rapport de partenariat qui lie l'ACE aux collectivités locales. Il précise que le premier possède la compétence artistique alors que les localités incarnent la légitimité issue des urnes et la connaissance du terrain :

Good partnerships develop when each side contributes something unique. Councils bring their local knowledge, broad range of responsibilities, cultural development work and, above all, their democratic representation. The Arts Council, on the other hand, brings expertise in the arts, experience of development in many contexts and a national perspective on local issues. ${ }^{36}$

Face à cette fronde, le ministre du DCMS, Chris Smith, a mis la réforme en attente. Nommée à sa place après les élections de 2001 durant lesquelles il avait été question de supprimer le DCMS comme l'Arts Council, Tessa Jowell a été chargée de la faire aboutir $^{37}$. 


\section{Mise en place et conséquences de la réforme de l'Arts Council of England}

21 La réforme a été votée en 2002 pour être appliquée durant la période d'intérim qui s'achevait en $2004^{38}$. Le gouvernement promet d'améliorer la rentabilité économique et sociale ('Value for money') des fonds de l'Arts Council en modernisant sa structure et ses méthodes de travail (contrats, audits...) ${ }^{39}$. L'organisme est constitué de trois parties : un bureau national à Londres, neuf agences régionales et un département qui se charge des services communs (ressources humaines, recrutement, finances) ${ }^{40}$. Tout en conservant l'objectif initial de modernisation, le gouvernement a pris en compte les critiques émanant des régions. On peut aussi y voir une réaction au débat sur l'utilisation des fonds de la loterie nationale et une réponse à la demande, impossible à satisfaire, de certains joueurs qui souhaitaient désigner le bénéficiaire de leur bulletin de jeu ${ }^{41}$. Le premier objectif était de réaliser des économies. L'ACE a évalué le coût de cette restructuration à 5,2 millions de livres pour l'année 2002-0342. Les économies en dépenses administratives devraient ensuite s'élever à 20 millions de livres sur trois ans (8 millions par la suite) et être redistribuées à la production artistique ${ }^{43}$. Le nombre total d'employés a été réduit d'environ un tiers grâce aux services désormais centralisés. Les structures des personnels ont été unifiées afin de pouvoir déléguer les mêmes tâches à toutes les agences régionales ${ }^{44}$. The Sunday Telegraph, non sans hypocrisie étant donné la position des conservateurs sur le sujet, regrette que l'organisme soit réduit à la "portion congrue » et que le pouvoir passe aux régions ${ }^{45}$. C'est à l'inverse en faveur de la décentralisation que le quotidien se positionne quelques mois plus tard: 'most assume that any funding changes will not help the arts. The last set of reforms [...] centralised funding control, reducing money available' ${ }^{\prime 46}$.

Le DCMS désirait également que l'Arts Council modernise ses méthodes de travail tout en étant plus à l'écoute des usagers. L'ACE et ses agences régionales ont publié des manifestes suivis de plans d'action. Le système des subventions a été rationalisé et simplifié. Un dossier unique de demande de subvention, appliquant les mêmes critères à toutes les régions, a remplacé la centaine qui existait auparavant, dont le Touring Programme et le Regional Arts Lottery Programme, créés pour attirer des fonds en dehors de Londres. L'ACE s'engage à traiter les dossiers dans un délai record et à renoncer au jargon administratif pour améliorer l'accès de tous aux subventions : 'The application [...] gives you the flexibility to describe your project or activity in your own words' ${ }^{\prime 47}$. L'Arts Council a augmenté de $200 \%$ son allocation au Voluntary Arts Network entre 2002-03 et 2005-06 et finance davantage d'organisations vouées à la pratique $\operatorname{artistique}^{48}$. Le discours de l'ACE présente cette nouvelle répartition des fonds comme un gage de démocratie culturelle ${ }^{49}$. Les publications de l'Arts Council décrivent la réforme comme radicale, ouvrant la voie vers un organisme moderne, progressiste, dynamique ${ }^{50}$. On perçoit dans ce discours des échos de la rhétorique du DCMS, ce qui tendrait à corroborer la thèse selon laquelle, malgré le principe d'autonomie, l'influence des gouvernements sur l'organisme s'accroît avec le temps et les nominations aux postes clés, comme on a pu le remarquer pendant la période conservatrice, entre 1979 et 1997. En 2003, lorsque Gerry Robinson quitte l'ACE après un mandat stratégique, il semble avoir convaincu une majorité des acteurs du bien fondé de la réforme. The Guardian le décrit comme l'un des présidents "les plus tenaces » ${ }^{51}$ qu'ait eu l'Arts Council. Selon Nigel Pittman, directeur de l'agence ACE de Londres, la 
réforme instaure un système cohérent qui conserve le caractère distinctif de chaque région tout en les incluant dans les décisions politiques nationales par leur représentation au Consei ${ }^{52}$. Bien comprise, cette réforme devrait donc limiter le rapport hiérarchique entre pouvoir national et pouvoir régional.

Le bilan de la réforme reste cependant à faire. Ainsi, en 2002-03, le bureau national de l'ACE dépensait encore 143 millions de livres sur les 258 millions disponibles pour l'ensemble de l'Angleterre. À partir de 2003-04, néanmoins, l'ACE s'est engagé à financer les bureaux régionaux proportionnellement à leur poids démographique et à décentraliser la majorité des responsabilités de financement et de gestion des organisations régionales ${ }^{53}$. L'organisme a montré des réticences à divulguer le budget national qui finance les tournées et les projets de dimension nationale avant la publication du rapport 2003-04. L'équilibre des pouvoirs entre les neuf directeurs régionaux et les six autres membres du Conseil ne transparaît pas encore ${ }^{54}$. L'ACE se définit désormais comme "une grande famille unie », expression qui entérine cependant la fin de l'indépendance des régions ${ }^{55}$.

\section{La décentralisation au profit des localités : imposée par le haut ou fruit d'un dialogue?}

24 À Preston, comme dans nombre de villes anglaises, le département loisir de la mairie fut créé dans les années soixante-dix au grand dam des conservateurs, qui redoutaient que les fonds de la ville ne soient engloutis en coûts administratifs et en construction de monuments « de style empire $»^{56}$. Aujourd'hui, en Angleterre, le niveau local est le deuxième financier des arts après l'Arts Council, soit directement soit par les fonds versés au bureau régional de l'ACE ${ }^{57}$. Tony Blair a déclaré vouloir mettre la culture au centre de son projet, et les localités travaillistes l'ont suivi. Ainsi, entre 1998 et 2002, les collectivités locales ont dépensé $56 \%$ de plus dans les galeries d'art contre seulement $4 \%$ d'augmentation en faveur du sport et des activités de loisir (recreation) ${ }^{58}$. La modernisation du service public n'épargne plus le secteur public de la culture. Le DCMS encourage et encadre les actions avec l'objectif d'intégrer l'intervention culturelle à la modernisation du gouvernement local. Quarante Local Public Service Agreements incluent des objectifs dans ce domaine. Depuis 1999, les collectivités locales sont tenues de rédiger un document exposant leur stratégie culturelle ${ }^{59}$. Celle de Londres reprend quatre dimensions essentielles de la politique nationale : modernisation, rhétorique, consultation, partenariats ${ }^{60}$.

Les directives du DCMS aux localités expriment les priorités générales du gouvernement à l'aide d'une rhétorique fondée sur des mots clés tels que : 'inclusion', ' access', 'diversity' et 'voice' ('give people a voice'). Le discours s'appuie également sur l'utilisation du terme "vision », introduit dès 1997 par Chris Smith, alors ministre du $\mathrm{DCMS}^{61}$. Cette rhétorique restait d'actualité en 2003 comme en attestent les paroles des conseillers travaillistes à la mairie de Preston: 'we have a vision for Preston'62. Cette "vision » implique un réveil de la culture au sens large et lie donc fortement les arts aux politiques de régénération urbaine, en y ajoutant une dimension de régénération sociale et citoyenne. C'est dans un sens plus traditionnel qu'on trouve le terme utilisé à Londres où 'The Mayor's Vision for London' introduit ses positions dans les différents domaines sous sa responsabilité, dont la culture ${ }^{63}$. La définition même du concept de culture a connu une réévaluation dans le discours travailliste qui lui donne un sens 
large, synonyme de mode de vie. À Preston, les attributions du département dont elle dépend actuellement sont révélatrices de ces évolutions : Regeneration, Community and Leisure Services. C'est un des départements les plus stratégiques de la mairie, qui s'est engagée dans une vaste entreprise de régénération urbaine. Le seul terme propre à la rhétorique artistique est celui d' "excellence ", qui est cependant compris au sens large de projet réussi, preuve de la distance qui a été prise vis-à-vis des critères de valeur appliqués à la culture d'élite ${ }^{64}$.

\section{Consultation et participation du citoyen : artifice ou réalité ?}

Les techniques utilisées pour améliorer les services publics sont parfois difficiles à appliquer au domaine de l'art subventionné, qu'il s'agisse d'études de marché, de consultation ou à plus forte raison de participation citoyenne. Les villes se heurtent à trois écueils spécifiques à la culture : le manque d'intérêt pour ce service public, la difficulté d'évaluer les besoins locaux et l'absence de techniques satisfaisantes de consultation et de participation des usagers.

Une consultation locale sur la programmation des arts du spectacle traditionnels se justifie rarement car elle concerne un public homogène, facile à connaître, contrairement à l'urbanisme qui concerne un large public. Les compagnies subventionnées doivent intégrer les objectifs de l'ACE : qualité, accessibilité, diversité. Pour les théâtres municipaux qui reçoivent des productions de l'extérieur, la difficulté réside dans l'équilibre de la programmation. Il faut offrir suffisamment de diversité dans l'offre tout en remplissant les salles, et essayer de comprendre les raisons des échecs (excès d'offre, mauvaise programmation...). Le théâtre municipal de Preston reçoit des troupes locales mais s'enorgueillit aussi d'entretenir un lien privilégié avec le Liverpool Philharmonic, dont le public bénéficie d'un programme imprimé séparément dans une brochure de luxe.

Les consultations locales sur les musées et les galeries sont plus courantes. Le Harris Museum and Gallery de Preston attire plus de 180000 visiteurs par an. Ses collections d'art visent en priorité l'excellence alors que les expositions d'histoire locale s'adressent à un public varié en intégrant une forte dimension éducative et une valorisation des différentes communautés. Le musée cherche aussi à impliquer le public dans la préparation des expositions. Pour l'une d'entre elles sur l'histoire du textile, les habitants ont été nombreux à prêter des vêtements d'époque. Les liens du Harris Museum and Gallery avec le Victoria and Albert Museum ont permis d'allier excellence et accessibilité : un groupe de femmes d'origine indienne s'est ainsi rendu à Londres pour rédiger le guide de l'exposition. Les tentatives visant l'appropriation de ces lieux publics par le biais d'activités adaptées aux goûts de chacun doivent être conduites au niveau local et nécessitent temps et personnels. Les retombées escomptées incluent l'investissement citoyen par le sentiment d'appartenance à la ville et de légitimité conférée par la dimension symbolique du lieu. La rentabilité de ce type d'action est néanmoins difficile à mesurer.

29 La consultation ciblée est essentielle pour les actions de proximité qui ont pour vocation de renouer le lien social dans les quartiers au travers de l'activité artistique. À Preston, une association de community arts, Prescap, a pour vocation de donner une voix 
à des groupes exclus (personnes handicapées, minorités ethniques, chômeurs...). Elle a mis au point depuis 1984 un moyen de consultation direct avec ses clients (clients). Généralement, ces derniers doivent être à l'origine du projet qui sera mené à bien en collaboration avec un community artist dont l'objectif est d'atteindre la qualité maximale sans inhiber les participants. Les produits finis, souvent des montages photographiques ou des peintures, sont d'une qualité qui légitime les subventions, initialement du Regional Arts Board, puis de la ville et du comté.

30 À Preston, l'Arts Forum a été créé avec l'objectif, selon la mairie, de donner la parole aux usagers des services culturels. La ville, comme il est courant en Angleterre, se caractérise par le dynamisme de ses associations artistiques, mais compte peu de militants dans ce domaine. L'élue à la culture est fière d'avoir mis en place un forum mais avoue finalement ne pas savoir comment en faire un instrument de démocratie locale. Selon elle, il n'est pas représentatif car, bien que toutes les associations concernées soient invitées, la parole y serait accaparée par les groupes de pression des arts d'élite, qui font déjà entendre leurs vœux. L'une des demandes formulées concernait la création d'un cinéma d'art et d'essai. La mairie a inclus le projet parmi ses 101 objectifs culturels, sans doute pour apaiser les intéressés et mettre en valeur le forum, mais avoue ne pas vouloir lui donner suite ${ }^{65}$. Le Forum a donc pour l'instant peu d'influence sur les décisions locales. Autre exemple de consultation aléatoire, celle qui a eu lieu lors de la soirée célébrant la culture afro-antillaise (Black Month), organisée par des associations représentant l'éventail de cette culture: histoire, arts d'élite et populaire, coiffure, cuisine. L'employée chargée de la diversité culturelle à la mairie a fait remplir durant la soirée des questionnaires de satisfaction qui n'avaient aucune représentativité, ce qu'elle a admis. Force est de constater que le public était très réduit et peu mixte.

31 Les personnes actives dans les arts sont ainsi plus faciles à consulter pour les collectivités locales, mais elles ne sont pas représentatives des habitants de la ville et leurs propositions correspondent rarement aux bénéfices sociaux ou économiques que la majorité des localités attendent de leur soutien à la culture. Les métropoles, comme Liverpool, qui sera Capitale européenne de la culture en 2008, ont davantage de moyens et de raisons de satisfaire des intérêts purement artistiques. À Preston, les subventions reçues par les groupes d'art amateur sont soumises à davantage de conditions depuis la mise en place de nouveaux formulaires de demande par les community and voluntary services qui n'incluent pas la qualité artistique mais exigent des associations de s'engager à faciliter l'adhésion de nouveaux membres issus d'une minorité. Les groupes de théâtre ou de musique se trouvent embarrassés car ils prospectent rarement pour trouver des membres et reçoivent des subventions minimes. Perplexes, ils choisissent généralement la catégorie « femmes » car ce sont déjà des activités mixtes.

La réalité de Preston ne semble donc pas à la hauteur de la rhétorique de ses élus, bien que ces derniers soutiennent quelques initiatives réussies, comme Prescap. Les directives ministérielles aboutissent ainsi plus souvent qu'il n'y paraît à des simulacres de consultation. De façon générale, l'art public souffre du manque d'intérêt d'une majorité de la population. Le citoyen ne pèse que sur des projets ponctuels. Les méthodes de participation se limitent à la conduite de consultations. 


\section{La gouvernance en réseau}

33 Le modèle de gouvernance en réseau s'applique d'autant plus à la culture qu'elle a rarement été un domaine d'intervention en soi. Depuis 1946, la charte de l'Arts Council précise les trois fonctions de l'organisme : financer, conseiller et informer, et travailler en partenariat ${ }^{66}$. Les réseaux intégrant les arts sont néanmoins devenus plus nombreux et plus complexes, les responsabilités plus fragmentées, car la culture se voit rattachée à des domaines d'actions très variés. Cela explique que les décisions ne s'appuient plus uniquement sur des critères artistiques ou de service public ${ }^{67}$. Les Regional Cultural Consortiums ont été créés par le gouvernement de Tony Blair pour inscrire au niveau régional les objectifs du DCMS (culture, sport, tourisme, médias). Ils bénéficient de fonds minimes, leurs tâches étant de formuler une stratégie culturelle régionale et de faire circuler l'information entre les intéressés (gouvernement central et local, agences régionales, distributeurs de la loterie nationale) ${ }^{68}$. Leur action paraît pour l'instant peu efficace, ce que les conservateurs regrettaient avant les élections de 2001 : 'It is entirely unclear what useful purpose is served by the Regional Cultural Consortiums, which we will abolish'69.

La collaboration entre les agences de l'ACE et les localités a montré davantage de potentiel. Leur première conférence nationale annuelle s'est tenue en 2002: 'The partnership between local government and Arts Council England is the bedrock of the arts in England. [...] We invest between us over $£ 800$ million a year in creativity and support thousands of jobs. ${ }^{70}$ Elle a abouti à un accord de partenariat identifiant quatre priorités communes: 'creative economies, healthy communities, vital neighbourhoods and engaging young people ${ }^{\prime 71}$. On remarque que trois de ces priorités ne sont pas proprement artistiques mais utilisent les arts à des fins économiques ou sociales. Le texte reprend la rhétorique du DCMS. La gouvernance à plusieurs niveaux y est définie comme la disparition des rapports hiérarchiques entre les différents pouvoirs ${ }^{72}$ : 'We are equal partners in a joint enterprise to improve quality of life in every community. ${ }^{73} \mathrm{Ce}$ lien a été inscrit dans la réforme de l'ACE qui accorde un rôle plus important aux élus locaux au sein de ses bureaux régionaux. Les membres de ces partenariats sont-ils vraiment à égalité ou a-t-on affaire à une forme officielle de consultation issue d'un organe national ? Le plan de redressement des théâtres régionaux est soumis à des subventions stables des collectivités locales ${ }^{74}$. Était-ce la condition posée par l'ACE pour poursuivre une collaboration qui l'éloigne de ses objectifs artistiques?

Dans la gouvernance en réseau, qui trouve ses origines dans l'idéologie du localisme des années 1990, les groupes de pression et les associations sont des acteurs essentiels pour permettre le passage d'une démocratie d'en haut (overhead) à une démocratie plurielle (pluralist) $^{75}$. Il est apparu que l'implication du secteur associatif est mieux garantie lorsque la ville n'est pas son unique interlocuteur. Pour conférer davantage de légitimité aux projets de dimension régionale, les agences régionales de l'Arts Council et le comté équilibrent les pouvoirs de la ville en octroyant des subventions séparées. Est-ce pour autant un gage de démocratie ? On a parfois l'impression que ce sont ellesmêmes que ces instances légitiment lorsque leurs choix s'accordent, d'autant que les critères de subvention se recoupent largement, surtout lorsque la ville et le comté sont travaillistes.

36 Le lien entre la culture et l'économie fait apparaitre un quatrième acteur, le secteur privé. En 2003, Preston a inauguré une ambitieuse stratégie culturelle dont l'objectif 
principal est de soutenir le programme de régénération autour de "101 façons" d'améliorer la ville avant la prochaine fête de la guilde ${ }^{76}$ de 2012 : "placing culture and creativity at the heart of the wider economic social and environmental well being of England's newest city ${ }^{77}$. Le Preston Cultural Partnership inclut 22 organisations publiques, privées, et $\mathrm{du}$ secteur associatif ${ }^{78}$. Le directeur du département régénération, loisir et communauté soutient que le plan d'action a été rédigé au terme d'une large consultation et de recherches indépendantes qui lui ont permis de convaincre des entreprises participant à la réhabilitation de la ville de soutenir des projets d'art public.

La collaboration des différents acteurs bute cependant sur leurs intérêts divergents. Nous avons vu que l'Arts Forum est peu écouté. Le projet visant à transformer le Harris Museum and Gallery en un V\&A Preston constitue un autre exemple de collaboration difficile. Le responsable de la régénération à la mairie soutient cette option qui séduit les partenaires privés, alors que sa collègue responsable des arts et directrice du musée qualifie cette idée de "formule marketing ". Les arguments de cette dernière, fondés sur la muséologie, risquent de peser faiblement face aux retombées économiques escomptées. Malgré la tradition britannique de mécénat culturel, la gouvernance en réseau dans ce domaine fait donc ressortir de fortes divergences d'intérêts.

\section{Conclusion}

La décentralisation des fonds et des décisions en faveur des arts est ambiguë car elle s'est surtout faite au profit des agences régionales d'un Arts Council désormais unifié. Les ambitions des localités sont en effet souvent fragilisées par les contraintes financières et artistiques des projets, la difficulté de mobiliser la population et l'impossibilité de vérifier au moyen des techniques habituelles le rapport qualité prix de ce service public à part. Cela justifie pour certains l'existence des agences régionales. La collaboration des deux niveaux se développe néanmoins sous la pression du gouvernement. La mise en place d'un échelon régional élu, les English Regional Assemblies, s'il incorporait des experts, pourrait modifier les équilibres en présence, mais cette perspective parait désormais repoussée ${ }^{79}$.

Les effets à long terme de la décentralisation sont à peine décelables. Il semble qu'elle opère un rapprochement vers un public «consommateur » de culture publique, mais modifie le travail des émetteurs. Les artistes, en apparence sortis gagnants de la réforme de l'ACE, doivent plus souvent que par le passé répondre à une demande relayée par les localités ou les agences régionales de l'ACE. Or, on peut considérer que la genèse d'un projet constitue la première étape d'une démarche créatrice. L'art public va-t-il devenir un art de commande ? On peut se féliciter que le ministère exerce une pression sur les localités qui négligent les arts, mais les fonds sont orientés de telle façon qu'une ville menant une politique alternative n'obtiendrait pas nécessairement le soutien du nouvel Arts Council «décentralisé ". Il reste à voir si la décentralisation aidera la culture à s'imposer comme domaine d'action indépendant, bien que, comme le souligne Robert Hewison, 'it is difficult to legislate for creativity's0. 


\section{BIBLIOGRAPHIE}

ARTS COUNCIL ENGLAND/LOCAL GOVERNMENT ASSOCIATION. Local Government and the Arts : A Vision for Partnership. London: ACE, 2003, 25 p.

ARTS COUNCIL ENGLAND. Ambitions for the Arts : 2003-2006 Manifesto. London: ACE, 2003, p. 2.

ARTS COUNCIL ENGLAND. 2003 Annual Review. London: ACE, 2004, 156 p.

D’ANGELO, Mario \& VESPÉRINI, Paul. Politiques culturelles en Europe : régions et décentralisation culturelle. Strasbourg : Conseil de l'Europe, 2000, 156 p.

DOUSTALY, Cécile. « La politique culturelle britannique, entre particularisme et européanisation » in T. SANDU (dir.), Identités nationales, identité européenne, visibilité internationale, Paris : L'Harmattan, 2004, pp. 103-120.

GRAY, Clive. The Politics of the Arts in Britain. London: Macmillan, 2000, 224 p.

GREEN, M. Issues and Problems in the Decentralizing of Cultural Planning : A Report for the UNESCO. Birmingham: CCCS, 1977, $52 \mathrm{p}$.

HEISKANEN, Ikka. Decentralisation : Trends in European Cultural Policies. Strasbourg: Council of Europe, 2002, $63 \mathrm{p}$.

HEWISON, Robert. 'Cultural Policy' in SELDON A. (ed.), The Blair Effect. London: Little, Brown \& Company, 2001, pp. 535-554.

SMITH, Chris. Creative Britain. London: Faber \& Faber, 1998, 170 p.

VALDIN-GUILLOU, Alice. « Un Aspect de la politique culturelle en Grande-Bretagne : le rôle de l'Arts Council ». Les Cahiers Charles V, Université Paris VII, n³ 3, avril 1981, pp. 107-125.

\section{NOTES}

1. Les agences régionales de Resource et du NFC sont dotées de larges pouvoirs. S. FORRESTER \&

D. LLOYD, The Arts Funding Guide, $6^{\text {th }}$ edition, London: The Directory of Social Change, 2002, p. 8.

2. La culture, soutenue par les fonds européens en tant que domaine d'action privilégié de régénération urbaine, permet aux villes de trouver une certaine indépendance par rapport à l'échelon national. Ce pouvoir d'autonomie issu de la communauté européenne constitue une autre force de décentralisation que nous n'avons pas l'espace d'analyser ici. Voir C. DOUSTALY "La politique culturelle britannique, entre particularisme et européanisation », in T. SANDU (dir.), Identités nationales, identité européenne, visibilité internationale, Paris : L'Harmattan, 2004, pp. 103-120.

3. Cet article utilise les données de terrain recueillies en octobre 2003 (31 entretiens avec le personnel et les élus du Preston City Council et du Lancashire County Council, avec les membres et le personnel du North West Arts Board et avec les acteurs de la vie culturelle).

4. La ville compte 25000 étudiants et $10 \%$ des habitants sont issus de minorités ethniques d'origines variées (proportion légèrement plus élevée que la moyenne nationale). <http:// www.auditcommission.gov.uk/reports/BVIR.asp?> (10/10/2003).

5. <http://www.auditcommission.gov.uk/reports/BVIR.Asp?> (10/10/2003). Ces inspections Best Value portent sur le rapport qualité-prix (value for money) des services publics locaux. 
6. M. D’ANGELO \& P. VESPÉRINI, Politiques culturelles en Europe : régions et décentralisation culturelle, Strasbourg: Conseil de l'Europe, 2000, p. 46.

7. A. VALDIN-GUILLOU, «Un Aspect de la politique culturelle en Grande-Bretagne : le rôle de l'Arts Council », Les Cahiers Charles V, Université Paris VII, n³, avril 1981, p. 120.

8. M. GREEN, Issues and Problems in the Decentralizing of Cultural Planning: A Report for the UNESCO, Birmingham: CCCS, 1977, pp. 41-43.

9. I. HEISKANEN, Decentralisation : Trends in European Cultural Policies, Strasbourg: Council of Europe, 2002, p. 7.

10. M. GREEN, op. cit., pp. 45-46 et F. MATARASSO \& C. LANDRY, Balancing Acts, 21 Strategic Dilemmas for Culture, Strasbourg: Council of Europe, 1999, pp. 47-49.

11. F. MATARASSO \& C. LANDRY, op. cit., p. 48.

12. S. FORRESTER \& D. LLOYD, op. cit., p. 7.

13. R. BROOK, 'The Good, the bad and the Arts Council', The Sunday Times, 02/11/2003.

14. La province se voyait pourvue en bibliothèques et musées, associés à l'éducation de masse, souci hérité de la tradition municipale victorienne. M. GREEN, op. cit., pp. 10-13.

15. Les références de ce paragraphe et du suivant sont tirées de : C. GRAY, The Politics of the Arts in Britain, London: Macmillan, 2000, pp. 136-138, p. 168, et pp. 175-176 ; M. GREEN, op. cit., pp. 41-43, A. VALDIN-GUILLOU, op. cit., p. 121.

16. GULBENKIAN FOUNDATION \& Lord REDCLIFFE-MAUD, Support for the Arts in England and Wales, London: T\&A Constable Ltd, 1976, p. 37.

17. 'decentralisation, not devolution' in ARTS COUNCIL OF GREAT BRITAIN, Thirty-Second Annual Report and Accounts. Value For Money. 1976-77, London: ACGB, 1977, p. 15.

18. C. GRAY, op. cit., p. 137.

19. A. VALDIN-GUILLOU, op. cit., p. 120.

20. La décentralisation ne risque-t-elle pas alors d'être limitée à une passation de pouvoir des groupes de pression nationaux à leurs équivalents locaux? M. THATCHER, The Downing Street Years, London: Harper Collins, 1993, p. 632.

21. C. GRAY, op. cit., p. 164. Le Greater London Council siégea de 1981 à 1986.

22. <www.artscouncil.org.uk/regions/information-detail/> (06/10/04).

23. Charlotte HIGGINS, 'For a few pennies more', The Guardian, 28/10/2002.

24. Les subventions ont augmenté de 186 à 410 millions de livres en 8 ans. Néanmoins, en 2002, le gouvernement central français dépensait en moyenne par habitant pour l'art le double de son équivalent anglais. <www.artscouncil.org.uk/historyoftheartscouncil/> (09/08/2004).

25. Le Dôme de la connaissance, musée des innovations, était un grand projet des célébrations du millénaire. Lancé par les conservateurs, il s'est transformé en gouffre financier pour l'administration Blair, qui a accepté de le reprendre. R. HEWISON, 'Cultural Policy', in A. Seldon, (ed.), The Blair Effect, London: Little, Brown \& Company, 2001, pp. 546-550.

26. Charte royale du $1^{\mathrm{er}}$ avril 1994. Le DCMS ne gère plus les politiques artistiques de ces nations, bien qu'il conserve la responsabilité des médias et de la loterie nationale.

27. M. GREEN, op. cit., p. 30.

28. EQUITY, Theatre Funding Conference Report, London: Equity, 2000, pp. 31-32.

29. Maev KENNEDY, 'Frayling appointed to lead the Arts Council', The Guardian, 06/12/2003.

30. S. FORRESTER \& D. LLOYD, op. cit., p. 8.

31. Amanda HALL, 'The Gospel according to Gerry', The Guardian, 31/03/2003.

32. <www.artscouncil.org.uk/regions/information-detail/> (06/10/04).

33. Le projet de réforme s'intitulait adroitement 'working together for the arts' (ACE, 2001).

34. <www.artscampaign.org.uk/membersonly/> (02/08/2003).

35. 'Mayor and Assembly say no to new centralised Arts Board', 15/03/2001, in $<$ www.london.gov.uk/view_press_release $>(01 / 10 / 02)$. 
36. ARTS COUNCIL ENGLAND / LOCAL GOVERNMENT ASSOCIATION, Local Government and the Arts : A Vision for Partnership, London: ACE, 2003, p. 9.

37. N. LEBRECHT, 'The Red Baroness swoops in', The Daily Telegraph, 27/06/2001.

38. Elle est consignée dans la Supplementary Royal Charter du 31 Mai 2002 : 'Give effect to new arrangements for regionalisation of its structure and activities [...], powers to establish and to delegate powers and functions to regional councils.' In ACE, 2003 Annual Review, London: ACE, 2004, p. 41 et p. 84.

39. La création en 1999 de la Quality, Efficiency and Standards Team dont la vocation était de mesurer la qualité des services des organismes subventionnés par le DCMS, préfigurait cette évolution.

40. C. MILNER, 'Arts Council to cut a third of its staff', The Sunday Telegraph, 30/06/2002.

41. Soit 28 pence par livre. Zoe WILLIAMS, 'Life's a Lotto', The Guardian, 15/10/2002.

42. Dont 2,2 millions ont été financés par la loterie nationale. Le budget de l'ACE est constitué à $37,5 \%$ de fonds de la loterie, le reste du DCMS (2002-03). ACE, 2003 Annual Review, op. cit., p. 88, p. 135.

43. ACE LONDON, Newsletter $n^{\circ} 1$, op. cit., p. 2.

44. ARTS COUNCIL ENGLAND, 2003 Annual Review, op. cit., p. 41.

45. 'a wafer thin organisation'. In C. MILNER, op. cit. The Sunday Telegraph, 30/06/2002.

46. The Daily Telegraph, 'news', 02/01/2003.

47. ARTS COUNCIL ENGLAND, 2003 Annual Review, op. cit., p. 127.

48. <www.artscouncil.org.uk/pressreleases>, 'Arts enter new era of growth', 25/03/2003.

49. Le large plan de redressement des théâtres n'a pourtant concerné que $15 \%$ des 500 théâtres affiliés à l'Independent Theatre Council et s'est concentré sur les grandes métropoles régionales (Bristol, Manchester). Alfred HICKLING, 'Local shows for local people', The Guardian, 12/11/2003.

50. ARTS COUNCIL ENGLAND, Ambitions for the Arts : 2003-2006 Manifesto, London: ACE, 2003, p. 2.

51. 'one of the toughest holders of the office' in Maev KENNEDY, 'Frayling appointed to lead the Arts Council', The Guardian, 06/12/2003.

52. ACE LONDON, Newsletter $n^{\circ} 1$, London: ACE, London, April 2004, p. 8.

53. < www.artscouncil.org.uk/regions/information-detail> (06/10/2004) et ARTS COUNCIL ENGLAND, 2003 Annual Review, op. cit., p. 34, p. 38 et p. 53.

54. Entretien avec Loretta Okoh, ACE information officer, 10/10/2004.

55. Maev KENNEDY, 'Streamlined Arts Council foresees happier days', The Guardian, 13/02/2003.

56. $C^{\text {or }}$ Joe Hood, in C. DAMP, 'Leisure Chief is a Waste of Money', Lancashire Evening Post, 8/02/1974. Le gouvernement central faisait pression sur les localités pour qu'elles créent de tels départements, notamment pour combattre la délinquance juvénile.

57. DEPARTMENT FOR CULTURE, MEDIA AND SPORT ARTS DIVISION, Guide to Arts Funding in England, London: DCMS, 2003, p. 6. En 2001-02, le total versé par les localités du Nord-Ouest à son RAB s'élevait à 764000 livres (7 livres par habitant en moyenne) contre 21000000 livres provenant d'organismes nationaux. In North West Arts Board, Report 2001-02, Manchester: NWAB, 2002, pp. 34-36.

58. Key Note 2003, Local Government services, Hampton: KNMR, 2003, p. 128.

59. DEPARTMENT FOR CULTURE, MEDIA AND SPORT, Creating opportunities - Guidance for Local Authorities in England on Local Cultural Strategies, London: DCMS, 1999, 48 p.

60. Cette stratégie s'ajoute à celles des localités londoniennes. R. CHRISTIANSEN, 'The arts column : where's the beauty in Ken's cultural strategy?', The Daily Telegraph, 05/02/2003.

61. 'a vision for the arts', in C. SMITH, Creative Britain, op.cit., pp. 42-47.

62. V. Afrin, conseillère travailliste responsable du portefeuille Regeneration, Community and Leisure à la mairie de Preston (10/2003).

63. <www.london.gov.uk/londonissues/> (02/09/2004). 
64. Lire à ce sujet le chapitre 'Relativism' sur la fin du consensus concernant les critères de jugement appliqués à l'art in R. HOGGART, The Way We Live Now, London: Pimlico, 1996, 352 p.

65. Entretiens avec le personnel et les élus du Preston City Council, octobre 2003.

66. ARTS COUNCIL ENGLAND, 2003 Annual Review, op. cit., p. 28.

67. C. GRAY, op. cit, pp. 170-172.

68. À Londres, le Cultural Consortium se nomme Cultural Strategy Group. Il a été établi en 2000. S. FORRESTER \& D. LLOYD, op. cit., p. 8.

69. <www.artscampaign.org.uk/membersonly/> (03/07/2004).

70. ARTS COUNCIL ENGLAND/LOCAL GOVERNMENT ASSOCIATION, op. cit., p. 2.

71. ARTS COUNCIL ENGLAND, 2003 Annual Review, op. cit., p. 29.

72. G. STOKER, op. cit., p. 20.

73. ARTS COUNCIL ENGLAND /LOCAL GOVERNMENT ASSOCIATION, op. cit., p. 9.

74. Brian LOGAN, 'We're in the money', The Guardian, 13/10/2002.

75. G. STOKER, Transforming Local Governance, from Thatcherism to New Labour, Basingstoke: Palgrave, 2004, p. 10, p.14, p. 17.

76. Preston, comme d'autres bourgs en Angleterre, a été autorisé par charte royale à créer une guilde (Merchant Guild) en 1328. Depuis 1542, la ville organise une grande fête pour sa guilde tous les vingt ans. La dernière remonte à 1992 et la prochaine se tiendra en 2012.

77. Preston City Council/Preston Strategic Partnership, Preston : A Cultural Strategy for England's Newest City, Preston: PCC, 2003, p. 7. Le secteur économique de la création (creative industries) emploie deux millions de Britanniques. DEPARTMENT FOR CULTURE, MEDIA AND SPORT, 2004 Annual Report, London: DCMS, p. 32.

78. Médias locaux, Chambre de commerce, transports, police, associations liées à la santé, à la religion, aux cultures minoritaires (associations de femmes, Council for Racial Equality).

79. L'échec massif ( $78 \%$ de voix contre) du référendum sur l'établissement d'une assemblée régionale dans le nord-est va certainement repousser le projet déjà fragilisé. H. MULHOLLAND, 'North-East voters reject regional assembly', The Guardian, 05/11/2004.

80. R. HEWISON, The Blair Effect, op. cit., p. 536.

\section{RÉSUMÉS}

La décentralisation du soutien public aux arts est généralement considérée comme un gage de démocratie culturelle. Les réformes de l'Arts Council et l'investissement accru des localités constituent-ils une réelle décentralisation alors que leur ministère encadre davantage leurs actions? Comment définir les rapports de ces acteurs que la rhétorique travailliste présente comme des partenaires égaux?

Decentralisation of public support for the arts is widely assumed to benefit cultural democracy. One may wonder whether Arts Council reforms and the increase in local authority funding really amount to decentralisation when the Ministry increasingly intervenes in their work. How can we define the links between these bodies which Labour rhetoric describes as equal partners? 
AUTEUR

CÉCILE DOUSTALY

Université Paris III 